\title{
Increased frequency of peripheral blood follicular helper $T$ cells and elevated serum IL-21 levels in patients with knee osteoarthritis
}

\author{
YUXING SHAN $^{1}$, CHANGLIN QI $^{1,2}$, YIJUN LIU $^{1}$, HUI GAO $^{1}$, DING ZHAO ${ }^{1}$ and YANFANG JIANG ${ }^{1,3,4}$ \\ ${ }^{1}$ Genetic Diagnosis Center, The First Hospital of Jilin University, Changchun, Jilin 130021; ${ }^{2}$ Department of Orthopaedics, \\ Shandong Jining No. 1 People's Hospital, Jining, Shandong 272000; ${ }^{3}$ Key Laboratory of Zoonosis Research, \\ Ministry of Education, The First Hospital of Jilin University, Changchun, Jilin 130032; ${ }^{4}$ Jiangsu Co-innovation Center for \\ Prevention and Control of Important Animal Infectious Diseases and Zoonoses, Yangzhou, Jiangsu 225009, P.R. China
}

Received November 20, 2015; Accepted November 18, 2016

DOI: $10.3892 / \mathrm{mmr} .2017 .6132$

\begin{abstract}
An aberrant immune response has been implicated in the pathogenesis of osteoarthritis (OA). However, the role of peripheral blood follicular helper T (TFH) cells in the pathogenesis of OA has yet to be elucidated. The purpose of the present study was to examine the role of TFH cells and serum interleukin-21 (IL-21) in the pathogenesis of OA. Frequency of peripheral blood inducible costimulator (ICOS)+, programmed death 1 (PD-1)+, and IL-21+CXCR5+CD4+ T cells in 40 patients with $\mathrm{OA}$ and 13 healthy controls (HCs) were examined by flow cytometry. The disease state in individual patients was assessed using the Western Ontario and McMaster Universities Osteoarthritis Index (WOMAC). Concentrations of serum IL-21, interferon- $\gamma$ (INF- $\gamma$ ), IL-4, IL-17A, and C-reactive protein (CRP) were determined, and the erythrocyte sedimentation rate (ESR) was measured. The percentages of CXCR5+CD4+ cells, PD-1+CXCR5+CD4+, ICOS+CXCR5+CD4+ and IL-21+CXCR5+CD4+ T cells in OA patients were significantly higher than those in the HCs. Furthermore, serum IL-21, IL-17A and IFN- $\gamma$ levels in OA patients were significantly higher than those in HCs. Expression of IL-21+TFH cells in OA patients demonstrated a positive correlation with OA disease activity, CRP levels and WOMAC. TFH cells and IL-21 appear to serve an important role in the progression of OA. IL-21+TFH cells may prove to be a marker of OA disease activity.
\end{abstract}

\section{Introduction}

Osteoarthritis (OA) is a degenerative and inflammatory disease of joints that affects an estimated $10 \%$ of men and

Correspondence to: Professor Yanfang Jiang, Genetic Diagnosis Center, The First Hospital of Jilin University, 71 Xinmin Street, Changchun, Jilin 130021, P.R. China

E-mail: yanfangjiang@hotmail.com

Key words: follicular helper T cells, interleukin-21, osteoarthritis, Western Ontario and McMaster Universities Osteoarthritis Index
$18 \%$ of women $>60$ years of age. The condition causes severe symptoms, including impaired mobility, joint deformities, and disability (1). Pathologically, OA is characterized by cartilage degeneration and osteophyte formation at the affected joints (2). Currently available treatment consists of pain management and joint replacement in patients with end-stage disease; however, therapies to control the progression of OA are in their early stages. In addition to the limited lifespan of prostheses, arthroplasty for osteoarthritic joints may be associated with adverse outcomes (3).

The causation of OA is thought to be multifactorial, with factors including age, body weight, gender, bone density, trauma and genetic susceptibility hypothesized to be involved (4). The pathogenesis of OA is not completely understood. Emerging evidence suggests the involvement of immunological factors in the development and progression of OA. Shen et al (5) demonstrated that CD4+T cells may serve a role in inducing inflammation in the early stages of OA, as well as being instrumental in causing inflammatory damage to the articular cartilage in the latter stages. According to Da et al (6), approximately half of all cases of OA manifest mild-to-moderate B lymphocytic infiltration in the synovial tissues, and the degree of B cell infiltration is directly correlated with the severity of local inflammation. Anti-cyclic citrullinated peptide (anti-CCP) antibodies have also been shown to be involved in the autoimmune processes of early-stage knee OA (7).

CD4+T cells, particularly $\mathrm{T}$ follicular helper $(\mathrm{TFH})$ cells, are known to regulate $\mathrm{B}$ cell activation and functional differentiation (8). Although the identification of TFH cells remains controversial, a previous study identified that CXCR5+CD4+ $\mathrm{T}$ cells shared the functional properties of $\mathrm{TFH}$ cells. Therefore, CXCR5+CD4+ T cells are considered to be TFH cells (9). Chemokine (C-X-C motif) receptor 5 (CXCR5), inducible costimulator (ICOS), programmed death (PD)-1, CD40 ligand, and the transcription factor, Bcl-6, are known to be expressed on the surface of TFH cells, and mediate the TFH cell-mediated activation of B cells within the lymphoid germinal centers $(10,11)$. Furthermore, interleukin-21 (IL-21), secreted by TFH cells, is known to modulate B cell differentiation and proliferation. In a previous study, increased levels of 
anti-CCP antibodies were demonstrated to be associated with a high frequency of TFH cells in patients with new-onset rheumatoid arthritis (RA) (12). Dysfunction of TFH cells and IL-21 is also known to be involved in the pathogenesis of systemic lupus erthymatosus and ankylosing spondylitis $(13,14)$. All these conditions are characterized essentially as chronic inflammatory joint diseases. However, the role of TFH cells in the pathogenesis of OA has yet to be fully elucidated.

The present study examined the frequency of peripheral blood TFH cells and the concentration of serum IL-21 in 40 patients newly diagnosed with OA and 13 healthy controls. The study also analyzed the frequency of different TFH cell subsets in the peripheral blood of patients with different grades of OA, and assessed the potential association with clinical characteristics. The present study was aimed at assessing the immunopathological roles and correlates of TFH cells in OA.

\section{Materials and methods}

Patients and controls. A total of 40 newly diagnosed OA patients were enrolled at the inpatient service of the First Hospital of Jilin University (Changchun, China) and 13 gender, age, and ethnicity-matched healthy controls were also recruited. The diagnosis of OA was made according to the clinical and radiographic criteria of the American College of Rheumatology (15). Knee radiographs were evaluated according to the Kellgren and Lawrence (KL) classification criteria (16). OA patients were defined as having radiographic knee OA of KL grade $\geq 2$ in at least one knee, whereas controls were having KL grades of 0 . None of the patients had been administered steroids, nonsteroidal anti-inflammatory drugs or other immunosuppressants one month prior to the blood sample collection. The severity of the disease in individual patients was measured using the Western Ontario and McMaster Universities Osteoarthritis Index (WOMAC) using a questionnaire containing three sections: i) Pain assessment (five criteria); ii) stiffness assessment (two criteria); and iii) functional assessment (seventeen criteria). Patients were rated against each criterion on a 5-point Likert Scale (0, none; 1, slight; 2, moderate; 3, severe; 4, extreme) (17). Patients with RA, traumatic arthritis, multiple sclerosis, type 1 diabetes, immune deficiency, chronic inflammatory diseases, and those with recent infection were excluded from the present study. The demographic and clinical characteristics of the study population are summarized in Table I. Written informed consent was obtained from all subjects. The study protocol was approved by the Ethics committee at the First Hospital of Jilin University (Changchun, China).

Laboratory examinations. Fasting venous blood samples $(10 \mathrm{ml})$ were obtained from individual subjects, and their sera were prepared by density-gradient centrifugation using Ficoll-Paque Plus at $468 \times \mathrm{g}$ for $15 \mathrm{~min}$ at $37^{\circ} \mathrm{C}$ (GE Healthcare Life Sciences, Uppsala, Sweden). The number of white blood cells (WBCs), erythrocyte sedimentation rate (ESR), and the concentration of serum C-reactive protein (CRP) were measured using Siemens special protein analysis instrument (Siemens AG, Munich, Germany).

Peripheral blood mononuclear cell (PBMC) stimulation. PBMCs were isolated by density-gradient centrifugation using
Table I. Demographic and clinical characteristics according to study group.

\begin{tabular}{lcc}
\hline & \multicolumn{2}{c}{ Group } \\
\cline { 2 - 3 } Variable & Healthy controls & OA \\
\hline Number of subjects (n) & 13 & 40 \\
Age (years) & $61(55-65)$ & $65(53-73)$ \\
Gender female, n (\%) & $10(76 \%)$ & $29(72 \%)$ \\
KL grade & & \\
II $(\%)$ & NA & $12(30 \%)$ \\
III $(\%)$ & NA & $15(37.5 \%)$ \\
IV $(\%)$ & NA & $13(32.5 \%)$ \\
WBC $(10 \% / 1)$ & $5.88(4.2-8.9)$ & $5.76(4.5-9.2)$ \\
ESR (mm/h) & $7(2-14)$ & $12(3-22)$ \\
CRP (mg/dl) & $1.5(1.3-2.7)$ & $2.31(0.79-5.14)^{\mathrm{a}}$ \\
Fibrae sanguis $(\mathrm{mg} / \mu \mathrm{l})$ & $251(150-378)$ & $265(211-496)$ \\
WOMAC & NA & $52(37-69)$ \\
Pain & NA & $12(8-15)$ \\
Stiffness & NA & $4(2-6)$ \\
Physical function & NA & $40(30-48)$ \\
& &
\end{tabular}

Data are expressed as the median (range) or as frequencies. ${ }^{\mathrm{a}} \mathrm{P}<0.05$ vs. healthy controls. Normal ranges of individual measures are CRP: 0-3 mg/dl; ESR: 0-15 mm/h, fibrae sanguis; 200-400 mg/ $\mu 1$ and WBC: (4-10)x109/1. KL, Kellgren and Lawrence; NA, not applicable; WBC, white blood cell count; ESR, erythrocyte sedimentation rate; CRP, C-reactive protein; WOMAC, Western Ontario and McMaster Universities Osteoarthritis Index; OA, osteoarthritis.

Ficoll-Paque Plus at $800 \times \mathrm{g}$ for $30 \mathrm{~min}$ at $37^{\circ} \mathrm{C}$ (GE Healthcare Life Sciences). PBMCs $\left(4 \times 10^{6}\right.$ cells $\left./ \mathrm{ml}\right)$ were cultured in RPMI-1640 medium with $10 \%$ fetal calf serum (Hyclone ${ }^{\text {TM }}$; GE Healthcare Life Sciences Waltham, MA, USA) in 24-well U-bottom tissue-culture plates (Corning Costar Inc., Corning, NY, USA). Cells were stimulated with or without $50 \mathrm{ng} / \mathrm{ml}$ phorbol myristate acetate and $2 \mathrm{~g} / \mathrm{ml}$ ionomycin (Sigma-Aldrich, St. Louis, MO, USA) at $37^{\circ} \mathrm{C}$ in a humidified incubator containing $5 \% \mathrm{CO}_{2}$ for $1 \mathrm{~h}$. Cells were cultured with Brefeldin A (10 g/ml; GolgiStop ${ }^{\mathrm{TM}}$; BD Biosciences, San Jose, CA, USA) for $5 \mathrm{~h}$, and subjected to intraplasmic staining and flow cytometric analyses.

Flow cytometry. Human PBMCs $\left(5 \times 10^{5}\right.$ cells/tube) were stained with PerCP/Cy5.5 anti-CXCR5 (cat. no. 562781), fluorescein isothiocyanate (FITC) anti-CD4 (cat. no. 555346), phycoeryrthrin (PE) anti-CD278 (cat. no. 557802), and Brilliant Violet 421 (BV421) anti-CD279 (cat. no. 562516) antibodies (BD Pharmingen, San Diego, CA, USA) at room temperature in the dark for $30 \mathrm{~min}$. Control staining was performed using FITC anti-IgG1 (cat. no. 556649), PE anti-IgG1 (cat.no.551436), PerCP/Cy5.5 anti-IgG1 (cat. no. 550795), and BV421 anti-IgG1 (cat. no. 562438) antibodies (BD Pharmingen). Cell gating was set to isolate CD4+ cells. The number of CXCR5+CD4+ (TFH) cells per sample was analyzed using FlowJo software version 7.6.2 (TreeStar, Ashland, OR, USA) (18). 
A

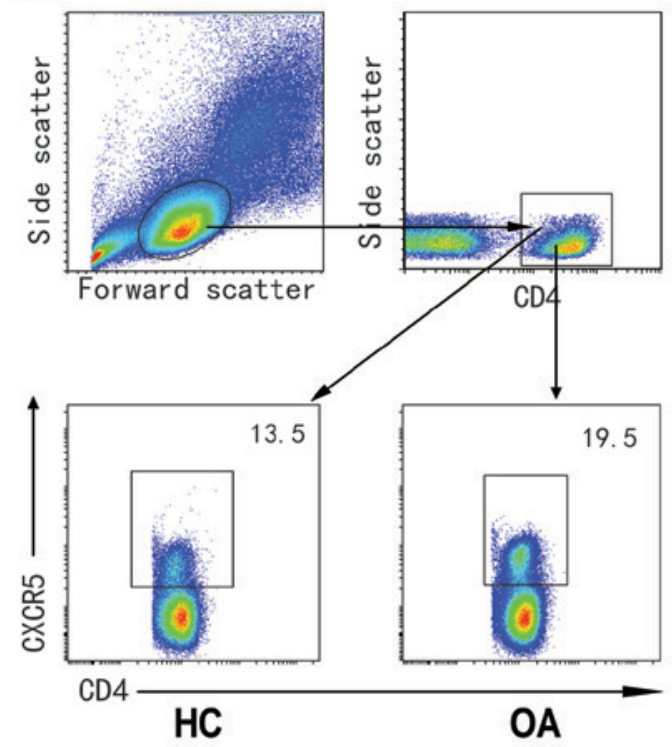

B

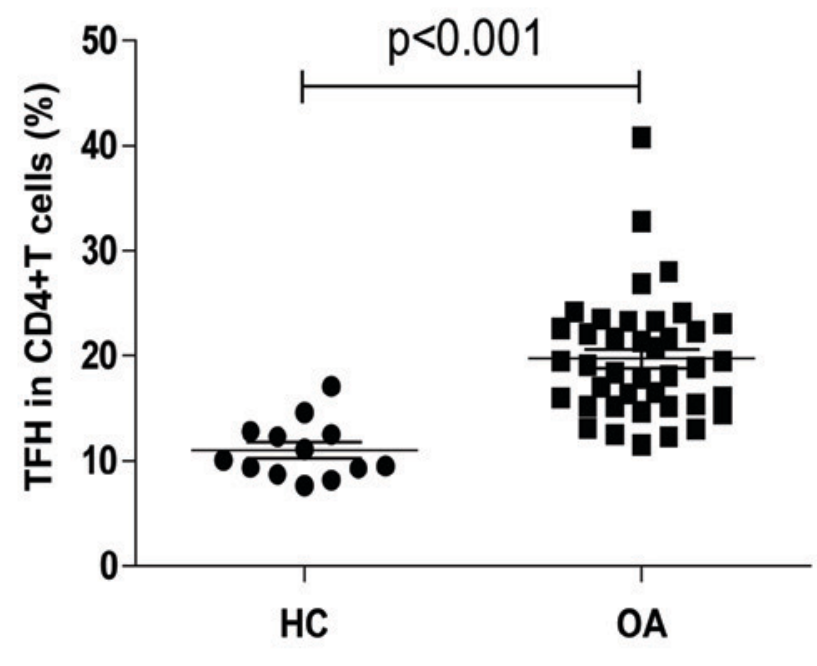

Figure 1. Flow cytometric analysis of the frequency of peripheral blood TFH cells. Peripheral blood mononuclear cells were isolated from individual patients and $\mathrm{HC}$, and stained with anti-CD4 and anti-CXCR5 or isotype controls. Subsequently, the cells were gated on living CD4+ cells, and the percentage of CXCR5+CD4+ TFH cells in total CD4+ T cells was determined. (A) Flow cytometric analysis. (B) Quantitative analysis. Data are expressed as the mean values of individual subjects from two separate experiments and the horizontal lines indicate the median values. OA group, All OA patients ( $\mathrm{n}=40$ ); HC group, healthy controls group $(\mathrm{n}=13)$. TFH, follicular helper $\mathrm{T}$; HC, healthy controls; OA, osteoarthritis; CXCR5, chemokine (C-X-C motif) receptor 5.

Stimulated PBMCs were harvested and stained simultaneously with PerCP/Cy5.5 anti-CXCR5 and FITC anti-CD4 antibodies at room temperature in the dark for $30 \mathrm{~min}$; the antibodies were diluted with TF Diluent Buffer (1:100; cat. no. 51-9008101; BD Pharmingen). Subsequently, cells were fixed, permeabilized, and stained with Alexa Fluor 647 anti-IL-21 antibody (BD Pharmingen). Percentages of IL-21+TFH cells were determined by flow cytometric analysis.

Cytometric bead array (CBA) analysis of serum cytokines. The concentrations of serum cytokines [IL-21, IL-4, IL-17A and interferon- $\gamma(\mathrm{INF}-\gamma)$ ] were determined using a CBA kit, according to the manufacturer's protocol (CBATM; BD Biosciences). Individual samples were quantified in duplicate on a fluorescence-activated cell sorting (FACS) Calibur cytometer (BD Biosciences), and the data were acquired using the CellQuestPro software, and subsequently analyzed using the CBA software (BD Biosciences) (19).

Statistical analysis. Data are expressed as the median and range, or as individual values. Inter-group differences were analyzed by the Mann-Whitney U nonparametric test with IBM SPSS software, version 19.0 (IBM SPSS, Armonk, NY, USA). The association between variables was evaluated using the Pearson rank correlation test. $\mathrm{P}<0.05$ was considered to indicate a statistically significant difference.

\section{Results}

Patient characteristics. The frequency of TFH cells in the blood samples obtained from 40 newly diagnosed OA patients and 13 HCs was assessed. No significant intergroup differences were observed in the distribution of TFH cells across age- and gender-matched subgroups.
Significantly higher levels of CRP were observed in OA patients compared with those in the $\mathrm{HCs}$, and considerable variability in WOMAC values was observed among OA patients (Table I).

High frequency of peripheral blood TFH cells in patients with $O A$. The frequency of peripheral blood CD4+cells was assessed using flow cytometry (Fig. 1A). No significant inter-group differences were observed in the frequency of CD4+T cells (data not shown). Percentages of CXCR5+CD4+TFH cells in $\mathrm{OA}$ patients were significantly higher than those in the $\mathrm{HC}$ group $(\mathrm{P}<0.001$, Fig. 1B).

Altered expression of TFH cell subsets in OA patients. Flow cytometry was also performed for quantitation of subsets of TFH cells (Fig. 1A). Significantly higher percentages of PD-1+CXCR5+CD4+, ICOS+CXCR5+CD4+ and IL-21+CXCR5+CD4+ T cells were observed in OA patients compared with those in $\mathrm{HCs}(\mathrm{P}<0.001, \mathrm{P}<0.001$, and $\mathrm{P}<0.001$, respectively; Fig. 2B-D). However, no significant difference was observed in the frequency of PD-1+ICOS+CXCR5+CD4+ $\mathrm{T}$ cells between the two groups (data not shown).

Frequency of different subsets of TFH cell in different grades of $O A$. To assess the potential association of different TFH cell subsets with progression of OA, TFH cell subsets were analyzed by different KL grades of OA and a higher frequency of TFH cells, and PD-1+ TFH cells in stage III OA were compared with those in grade II patients $(\mathrm{P}=0.03$ and $\mathrm{P}=0.002$, respectively; Fig. 3A and $\mathrm{B}$ ). In addition, no significant difference was identified in expression of the TFH cells and PD-1+ TFH cells between patients with advanced grade (III and IV) $\mathrm{OA}(\mathrm{P}=0.07$ and $\mathrm{P}=0.52$, respectively; Fig. $3 \mathrm{~A}$ and B). In addition, IL-21+TFH cells were significantly higher in 
A
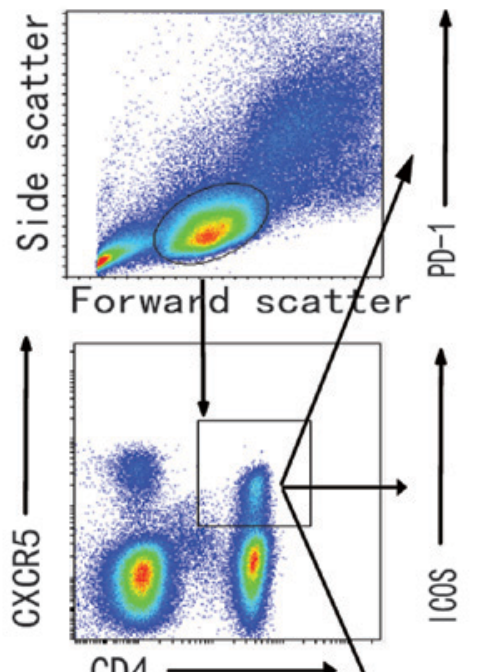

CD4
$\mathrm{HC}$
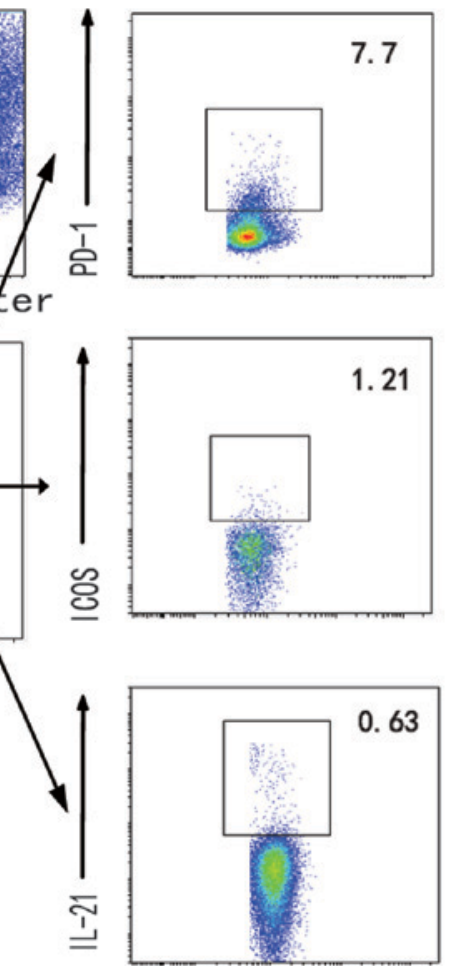

$\mathrm{OA}$

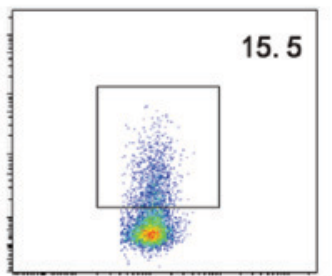

2. 44

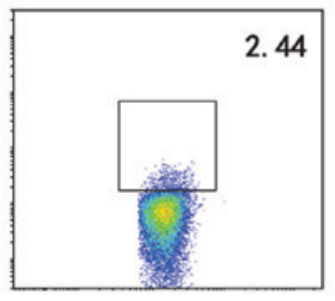

2. 05

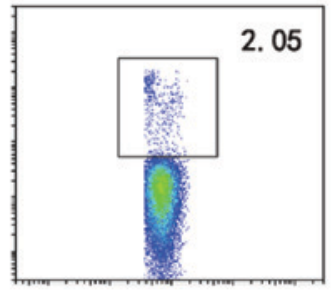

CD4

B

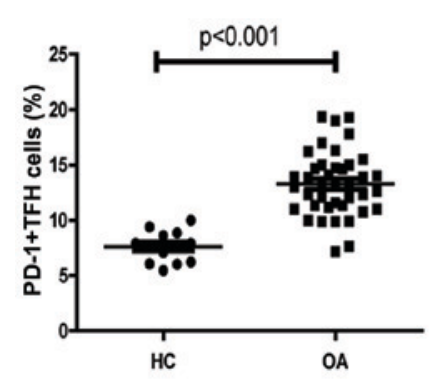

C

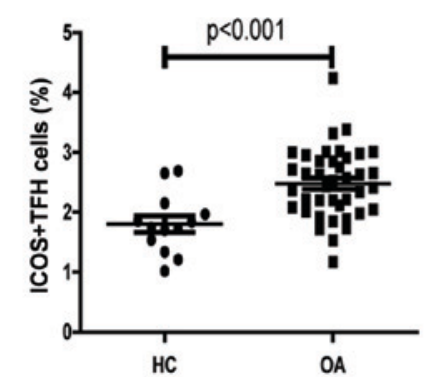

D

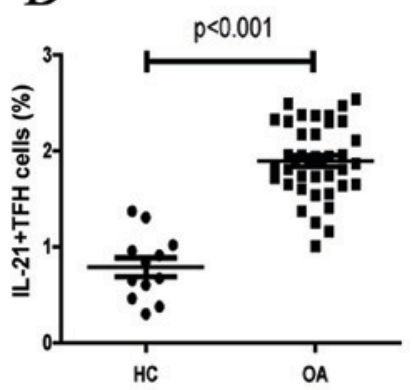

Figure 2. Flow cytometry for quantitation of subsets of TFH cells. Fluorescence-activated cell sorting analysis of the numbers of different subsets of TFH cells in individual subjects. Peripheral blood mononuclear cells were isolated from individual subjects and were stained in duplicate with anti-CD4, anti-CXCR5, anti-ICOS, anti-PD-1 and intracellular anti-IL-21 or isotype-matched IgG antibodies, respectively. The cells were characterized using flow cytometry with gating, initially on living lymphocytes, and then on CD4+CXCR5+TFH cells. Subsequently, the frequency of ICOS+, PD-1+ and IL-21+TFH in total TFH was analyzed, and a minimum of 30,000 events were analyzed for each sample. Data are expressed as the mean values of individual participants from two separate experiments. (A) Flow cytometry analysis. (B-D) The numbers of (B) CD4+CXCR5+PD-1+, (C) CD4+CXCR5+ICOS+ and (D) CD4+CXCR5+IL-21+TFH cells. The horizontal lines indicate the median values for each group. OA: All OA patients ( $\mathrm{n}=40)$, HC: healthy control group (n=13). TFH, follicular helper T; IL-21, interleukin-21; OA, osteoarthritis; (ICOS)+, inducible costimulator; (PD-1), programmed death 1; CXCR5, chemokine (C-X-C motif) receptor 5.

patients with stage IV disease compared with that in patients with stage III ( $\mathrm{P}=0.005$; Fig. 3C), in which the percentage of IL-21+ TFH cells was also significantly higher compared with that in stage II disease ( $\mathrm{P}=0.004$; Fig. 3C). Furthermore, the frequency of ICOS+TFH cells associated with different grades of OA were significantly higher compared with that in $\mathrm{HCs}$ $(\mathrm{P}=0.01$; Fig. 3D). However, no significant difference was observed in the frequency of ICOS+TFH cells between grades II and III, and between grades III and IV OA patients. $(\mathrm{P}=0.96$ and $\mathrm{P}=0.32$, respectively; Fig. 3D).

Variability in serum inflammatory cytokine levels in $O A$. Serum levels of the inflammatory cytokines, IL-21, IL-4,
IL-17A, and IFN- $\gamma$ were measured by CBA (Fig. 4). No significant differences in the levels of serum IL-4 were observed between $\mathrm{OA}$ patients and $\mathrm{HCs}(\mathrm{P}=0.07$, Fig. 4A). Furthermore, the concentrations of serum IL-21 $(\mathrm{P}<0.001$; Fig. 4B), IFN- $\gamma$ $(\mathrm{P}<0.001$; Fig. 4C) and IL-17A $(\mathrm{P}<0.001$; Fig. 4D) in $\mathrm{OA}$ patients were significantly higher than that in the HCs. Thus, increased levels of serum IL-21, IL-17A, and IFN- $\gamma$ may serve a crucial role in the development of OA.

Correlation of peripheral blood $I L-21+T F H$ cells with CRP level and disease severity. The potential association of the frequency of TFH cell subsets with clinical parameters and disease severity in OA patients was assessed using Pearson's 

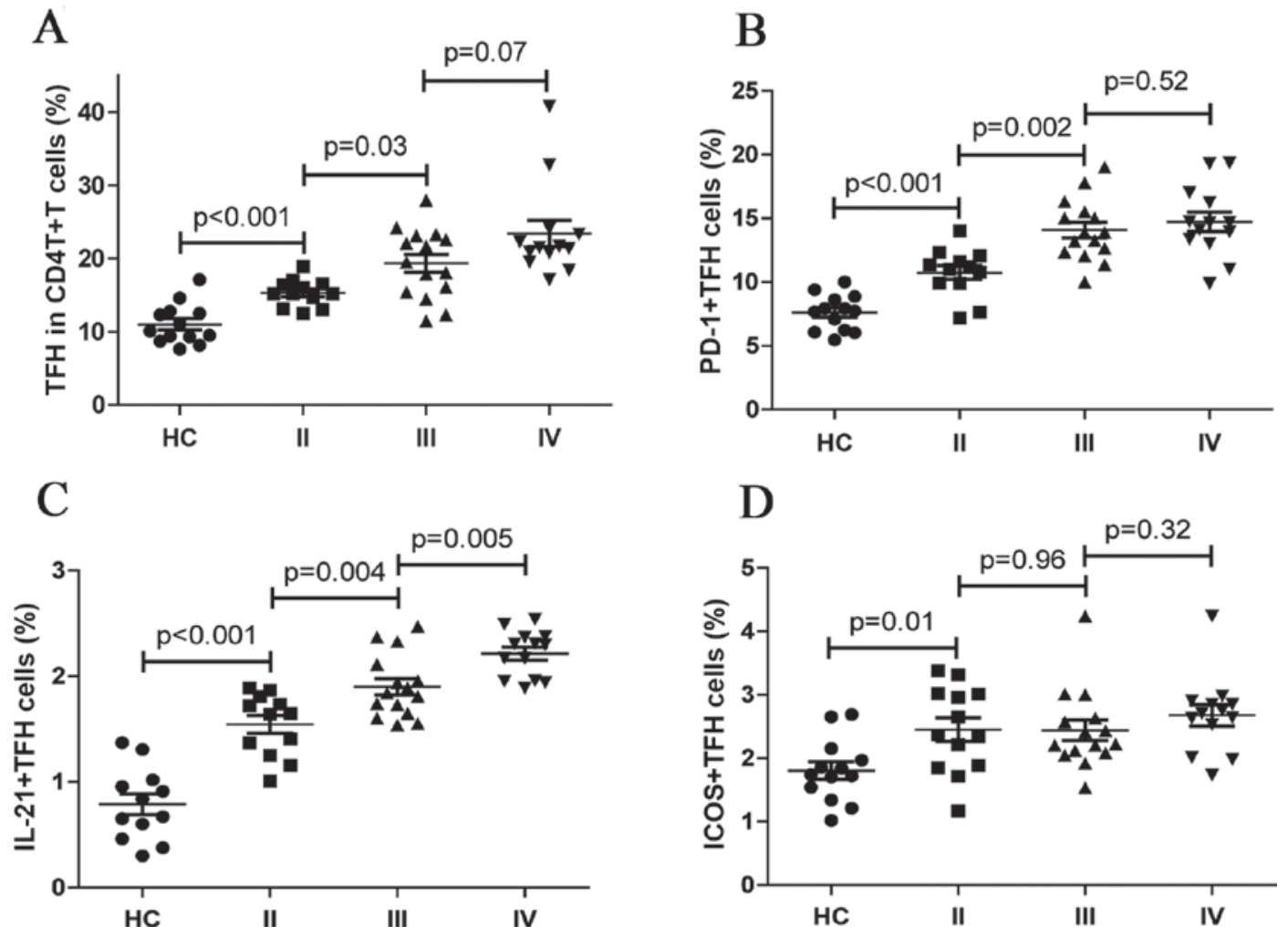

Figure 3. Quantification of peripheral blood TFH cell subsets disaggregated by OA clinical grade. The frequency of CD4+CXCR5+, PD-1+CD4+CXCR5+ IL-21+CD4+CXCR5+TFH and ICOS+CD4+CXCR5+ cells for stages II-IV of OA was analyzed. Knee radiographs were evaluated according to the KL classification criteria. Data are expressed as the mean values of individual participants from two separate experiments. (A-D) The frequency of (A) CD4+CXCR5+, (B) PD-1+CD4+CXCR5+, (C) IL-21+CD4+CXCR5+TFH and (D) ICOS+CD4+CXCR5+ cells in stage II-IV of OA. The horizontal lines indicate the median values for each group. OA patients with stage II $(n=12)$, OA patients with stage III $(n=15)$, OA patients with stage IV ( $n=13)$, and HC: healthy control group (n=13). OA, osteoarthritis; KL, Kellgren and Lawrence; IL-21, interleukin-21; PD-1, programmed death 1; TFH, follicular helper T.

correlation analysis. The percentage of IL-21+TFH cells correlated with CRP levels in OA patients $(\mathrm{R}=0.47, \mathrm{P}=0.002$; Fig. 5A); however, no significant correlation between TFH cells and the other clinical parameters was observed. The frequency of peripheral blood IL-21+TFH cells also demonstrated a positive correlation with WOMAC of OA patients $(\mathrm{R}=0.61, \mathrm{P}<0.001$; Fig. 5B). IL-21+TFH cells may be involved in the inflammatory state of OA, as they correlated with the symptoms and functionality score of OA patients.

\section{Discussion}

Although the pathophysiology of OA is poorly understood, immunological factors are widely acknowledged as serving an important role in the pathogenesis of OA. In the present study, the frequency of different subsets of circulating TFH cells was examined, and their association with the clinical characteristics of OA patients was evaluated. A significantly higher frequency of peripheral blood CXCR5+CD4+TFH cells was identified in patients with OA compared with that in HC. Previous studies have implicated CD4+T cells and B cells in the pathogenesis of OA (5-7). An imbalance of the immune response is known to be involved in the development of OA. TFH cells serve an important role in B cell differentiation, antibody production, and humoral immunity (8). Based on the findings of the present study, TFH cells appear to contribute to the development of an inflammatory environment that is characteristic of OA.
ICOS and PD-1 molecules are known to be expressed on the TFH cell surface $(10,11)$. Furthermore, ICOS+TFH cells are known to have a positive regulatory effect on humoral responses, whereas PD-1+TFH cells serve as negative regulators of TFH cell activity $(20,21)$. The frequency of the different subsets of circulating TFH cells was analyzed, and significantly higher percentages of ICOS+CXCR5+CD4+, PD-1+CXCR5+CD4+ and IL-21+CXCR5+CD4+T cells were identified in $\mathrm{OA}$ patients compared with those in HCs. However, no significant difference in the number of PD-1+ICOS+CXCR5+CD4+T cells was observed between the two study groups (data not shown). These findings appear to implicate activated TFH cell subsets in the development of OA. The increased frequencies of both PD-1+ and ICOS+TFH cells in the OA patients appear to be paradoxical, as these molecules have counter-regulatory effects on TFH cells. ICOS-mediated co-stimulation is crucial for TFH cell differentiation. PD-1+TFH cells may serve as negative regulators for the number and functionality of TFH cells, and to minimize collateral damage effected by the immune response. Notably, a similar phenomenon is also known to be associated with RA (22).

OA is characterized by cartilage and disc degeneration, and osteophyte formation at the joints (2). The morbid state in OA patients can be approximately categorized into different grades based on radiographic results according to the KL classification criteria (16). To characterize the altered dynamics of TFH cells in different grades of OA, the association of different 
A

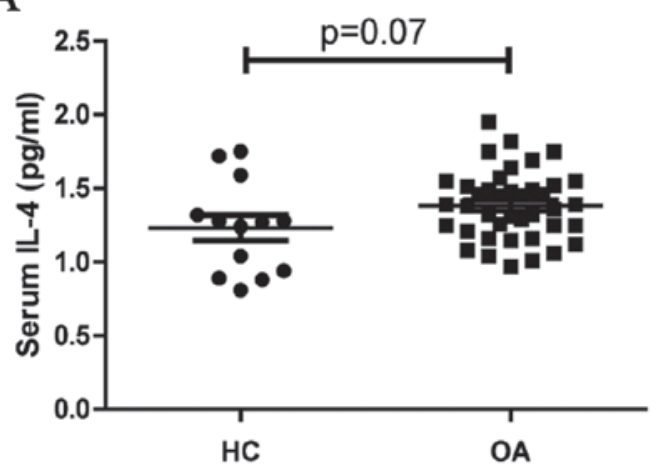

$\mathrm{C}$

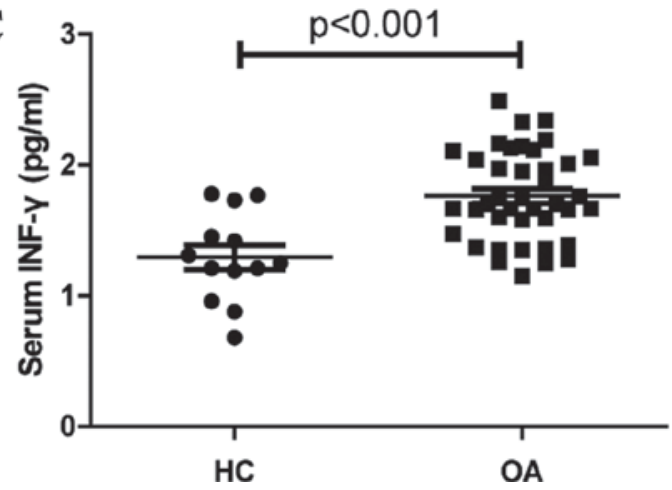

B

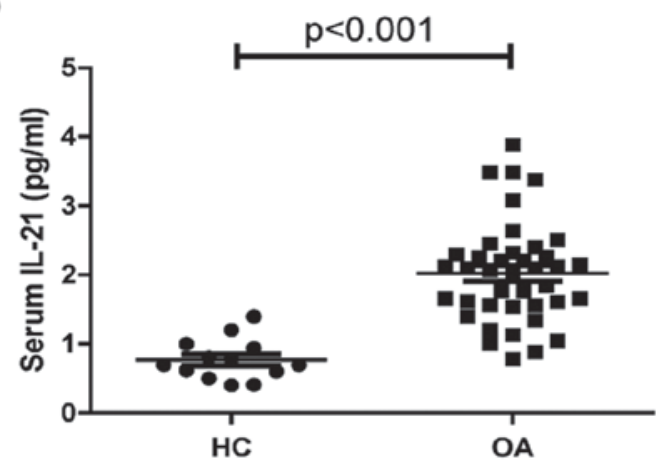

D

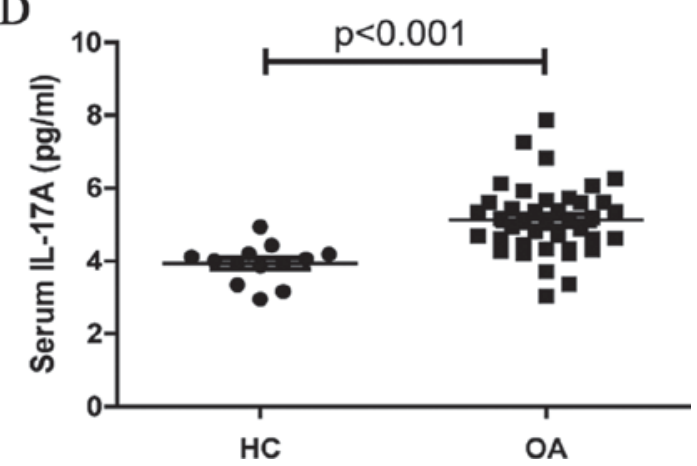

Figure 4. Analysis of serum cytokines. The concentrations of serum IL-21, IL-4, IFN- $\gamma$, and IL-17A in the HC and OA patients were determined by CBA. Data are expressed as the mean levels of serum (A) IL-4, (B) IL-21, (C) IFN- $\gamma$, and (D) IL-17A in the HC and OA patients. HC, healthy controls (n=13); OA, Osteoarthritis $(\mathrm{n}=40)$. IL-21, interleukin-21; HC, healthy controls; OA, osteoarthritis; CBA, cytometric bead array; IFN- $\gamma$, interferon- $\gamma$.

A

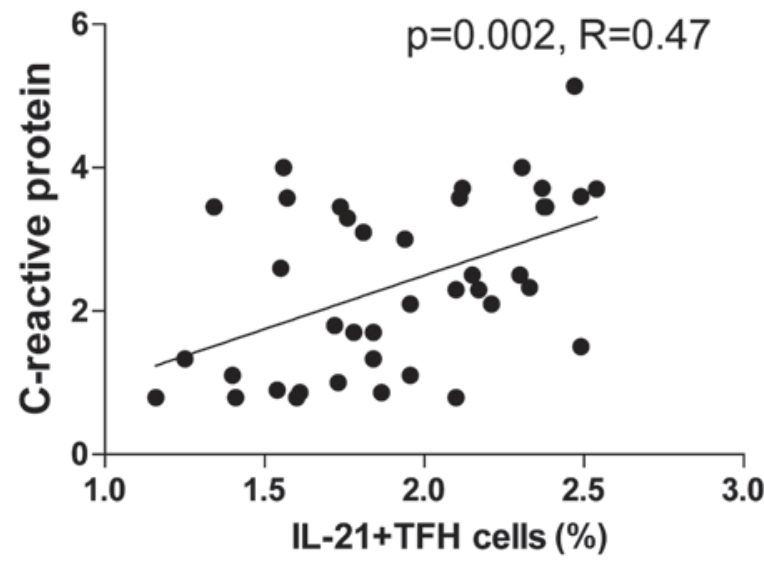

B

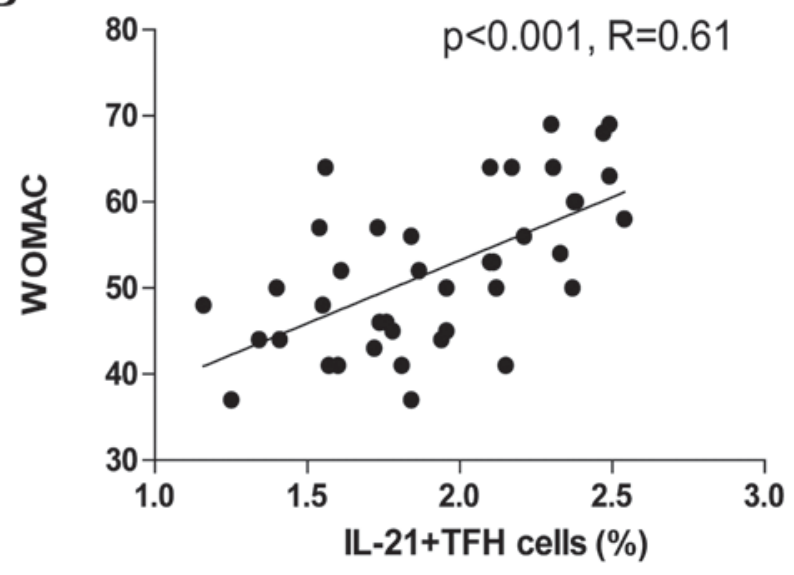

Figure 5. Correlation of IL-21+TFH cells with CRP levels, and correlation of IL-21+TFH cells with disease severity in OA. (A) Percentages of peripheral blood IL-21+TFH cells positively correlated with CRP, and (B) the frequency of peripheral blood IL-21+TFH cells positively correlated with WOMAC in OA patients. IL-21, interleukin-21; TFH, follicular helper T; CRP, C-reactive protein; OA, osteoarthritis; WOMAC, Western Ontario and McMaster Universities Osteoarthritis Index.

subsets of TFH cells with the clinical grade of OA was studied. The stage III-IV OA patients were identified to have higher frequencies of TFH cells and PD-1+TFH cells compared with those in stage II patients, whereas no significant differences with respect to TFH cells and PD-1+TFH cells between stage III and IV patients were identified. Furthermore, no significant differences in the expression of ICOS+TFH cells were observed between stage II, III and IV OA patients. Thus, it is possible that the high frequency of TFH cells may be a result of positive regulation mediated through ICOS in early-stage $\mathrm{OA}$, and the increased population of PD-1+TFH cells in the later stages may limit the TFH cell frequency as a negative regulator. Notably, a sustained increase in the numbers of IL-21+TFH cells was observed during disease progression, with high levels detected in the advanced stages of OA. Similarly, an increase in IL-21 levels was also observed in OA patients. Previous studies have reported elevated IL-21 levels and transcription product in the synovial fluid 
of both early- and advanced-stage knee OA $(23,24)$. Thus, IL-21+TFH cells appear to have an important role to serve in the progression of OA. The present study also identified higher levels of INF- $\gamma$ and IL-17 in OA patients. These results are consistent with those of previous studies, which indicated that OA was a T-helper cell 1 (Th1)-mediated form of arthritis (25). Yamada et al (25) and Lùrati et al (26) reported elevated levels of Th17 cells in OA patients, which further illustrated OA as a disease marked by an imbalance in the immune response towards a pro-inflammatory state.

Immunological factors serve an important role in $\mathrm{OA}$ pathogenesis (4-7). TFH cells are crucial regulators of $B$ cells, and IL-21 stimulates T and B cell proliferation $(8,10)$. IL-21 can increase the number of differentiated osteoclasts and induce bone marrow cells to differentiate into mature osteoclasts via upregulation of receptor activator of nuclear factor- $\kappa \mathrm{B}$ ligand (RANKL) expression (27). To study the association between TFH cells and OA, the correlation between different TFH cell subsets and WOMAC was analyzed. A positive correlation between the frequency of IL-21+TFH cells and WOMAC was observed in OA patients. Furthermore, a positive correlation was observed between IL-21+TFH cells and the CRP level, which is an inflammatory marker of OA patients. Based on these findings, IL-21+TFH cells appear to be involved in the inflammatory state of OA, and IL-21+TFH cells correlate with the symptoms and functionality score of OA patients, and therefore may serve as markers of OA disease activity.

The small sample size is a limitation of the present study, as is the lack of evaluation of the functional aspects of TFH cells. Therefore, further studies with larger sample sizes are required to elucidate the pathogenic mechanism of TFH cells.

In conclusion, a high frequency of CXCR5+CD4+TFH cells was observed in patients with OA, as compared with that in the HCs. Furthermore, a correlation between IL-21+TFH cells and CRP levels was observed. The findings of the current study indicate an association between IL-21+TFH cells and disease activity. IL-21+TFH levels may prove to be a useful marker of disease activity in OA patients. However, the exact mechanism of OA pathogenesis mediated by IL-21+TFH cells remains to be elucidated. In addition, further studies are required to determine whether IL21+TFH cells and IL-21 could be novel therapeutic targets for OA.

\section{Acknowledgements}

This study was supported by grants received from the National Natural Science Foundation of China (grant nos. 30972610 and 81273240), Jilin Province Science and Technology Agency (grant no. 20110716), the Health Department Research Projects in Jilin Province (grant no. 2009Z054) and the Norman Bethune Program of Jilin University (grant no. 2012206).

\section{References}

1. Woolf AD and Pfleger B: Burden of major musculoskeletal conditions. Bull World Health Organ 81: 646-656, 2003.

2. Guccione AA, Felson DT, Anderson JJ, Anthony JM, Zhang Y, Wilson PW, Kelly-Hayes M, Wolf PA, Kreger BE and Kannel WB: The effects of specific medical conditions on the functional limitations of elders in the Framingham study. Am J Public Health 84: 351-358, 1994.
3. Sharma L and Kapoor D: Epidemiology of Osteoarthritis. Osteoarthritis: Diagnosis and Medical/Surgical Management. 4th edition. Lippincott Williams \& Wilkins, Philadelphia, PA, 2007.

4. Sowers M: Epidemiology of risk factors for osteoarthritis: Systemic factors. Curr Opin Rheumatol 13: 447-451, 2001.

5. Shen PC, Wu CL, Jou IM, Lee CH, Juan HY, Lee PJ, Chen SH and Hsieh JL: T helper cells promote disease progression of osteoarthritis by inducing macrophage inflammatory protein- $1 \gamma$. Osteoarthritis Cartilage 19: 728-736, 2011.

6. Da RR, Qin Y, Baeten D and Zhang Y: B cell clonal expansion and somatic hypermutation of Ig variable heavy chain genes in the synovial membrane of patients with osteoarthritis. J Immunol 178: 557-565, 2007.

7. Du H, Masuko-Hongo K, Nakamura H, Xiang Y, Bao CD, Wang XD, Chen SL, Nishioka K and Kato T: The prevalence of autoantibodies against cartilage intermediate layer protein, YKL-39, osteopontin and cyclic citrullinated peptide in patients with early-stage knee osteoarthritis: Evidence of a variety of autoimmune processes. Rheumatol Int 26: 35-41, 2004.

8. Yusuf I, Kageyama R, Monticelli L, Johnston RJ, Ditoro D, Hansen K, Barnett B and Crotty S: Germinal center T follicular helper cell IL-4 production is dependent on signaling lymphocytic activation molecule receptor (CD150). J Immunol 185: 190-202, 2010.

9. Morita R, Schmitt N, Bentebibel SE, Ranganathan R, Bourdery L, Zurawski G, Foucat E, Dullaers M, Oh S, Sabzghabaei N, et al: Human blood CXCR5(+)CD4(+) T cells are counterparts of $\mathrm{T}$ follicular cells and contain specific subsets that differentially support antibody secretion. Immunity 34: 108-121, 2011.

10. Nurieva RI, Chung Y, Hwang D, Yang XO, Kang HS, Ma L, Wang YH, Watowich SS, Jetten AM, Tian Q and Dong C: Generation of $\mathrm{T}$ follicular helper cells is mediated by interleukin-21 but independent of T helper 1,2, or 17 cell lineages. J Immunity 29: 138-149, 2008.

11. Kerfoot SM, Yaari G, Patel JR, Johnson KL, Gonzalez DG, Kleinstein SH and Haberman AM: Germinal center B cell and $\mathrm{T}$ follicular helper cell development initiates in the interfollicular zone. J Immunity 34: 947-960, 2011.

12. Ma J, Zhu C, Ma B, Tian J, Baidoo SE, Mao C, Wu W, Chen J, Tong J, Yang M, et al: Increased frequency of circulating follicular helper T cells in patientswith rheumatoid arthritis. Clin Dev Immunol 2012: 827480, 2012.

13. Simpson N, Gatenby PA, Wilson A, Malik S, Fulcher DA, Tangye SG, Manku H, Vyse TJ, Roncador G, Huttley GA, et al: Expansion of circulating T cells resembling follicular helper $\mathrm{T}$ cells is a fixed phenotype that identifies a subset of severe systemic lupus erythematosus. Arthritis Rheum 62: 234-244, 2010.

14. Xiao F, Zhang HY, Liu YJ, Zhao D, Shan YX and Jiang YF: Higher frequency of peripheral blood interleukin 21 positive follicular helper $\mathrm{T}$ cells in patients with ankylosing spondylitis. J Rheumatol 40: 2029-2037, 2013.

15. Altman R, Asch E, Bloch D, Bole G, Borenstein D, Brandt K, Christy W, Cooke TD, Greenwald R, Hochberg M, et al: Development of criteria for the classification and reporting of osteoarthritis. Classification of osteoarthritis of the knee. Diagnostic and therapeutic criteria committee of the American rheumatism Association. Arthritis Rheum 29: 1039-1049, 1986.

16. Kellgren JH and Lawrence JS: Radiological assessment of osteo-arthrosis. Ann Rheum Dis 16: 494-502, 1957.

17. Bellamy N, Buchanan WW, Goldsmith $\mathrm{CH}$, Campbell $\mathrm{J}$ and Stitt LW: Validation study of WOMAC: A health status instrument for measuring clinically important patient relevant outcomes to antirheumatic drug therapy in patients with osteoarthritis of the hip or knee. J Rheumatol 15: 1833-1840, 1988.

18. Jiang Y, Ma Z, Xin G, Yan H, Li W, Xu H, Hao C, Niu J and Zhao P: Th1 and Th2 immune response in chronic hepatitis B patients during a long-term treatment with adefovir dipivoxil. Mediators Inflamm 2010: 143026, 2010.

19. Morgan E, Varro R, Sepulveda H, Ember JA, Apgar J, Wilson J, Lowe L, Chen R, Shivraj L, Agadir A, et al: Cytometric bead array: A multiplexed assay platform with applications in various areas of biology. Clin Immunol 110: 252-266, 2004.

20. Deenick EK and Ma CS: The regulation and role of T follicular helper cells in immunity. J Immunology 134: 361-367, 2011.

21. Rasmussen TK, Andersen T, Hvid M, Hetland ML, Hørslev-Petersen K, Stengaard-Pedersen K, Holm CK and Deleuran B: Increased interleukin 21 (IL-21) and IL-23 are associated with increased disease activity and with radiographic status in patients with early rheumatoid arthritis. J Rheumatol 37: 2014-2020, 2010 
22. Wang J, Shan Y, Jiang Z, Feng J, Li C, Ma L and Jiang Y: High frequencies of activated $\mathrm{B}$ cells and $\mathrm{T}$ follicular helper cells are correlated with disease activity in patients with new-onset rheumatoid arthritis. Clin Exp Immunol 174: 212-220, 2013.

23. Scanzello CR, Umoh E, Pessler F, Diaz-Torne C, Miles T, Dicarlo E, Potter HG, Mandl L, Marx R, Rodeo S, et al: Local cytokine profiles in knee osteoarthritis: Elevated synovial fluid interleukin-15 differentiates early from end-stage disease. Osteoarthritis Cartilage 17: 1040-1048, 2009.

24. Kapoor M, Martel-Pelletier J, Lajeunesse D, Pelletier JP and Fahmi H: Role of proinflammatory cytokines in the pathophysiology of osteoarthritis. Nat Rev Rheumatol 7: 33-42, 2011.
25. Yamada H, Nakashima Y, Okazaki K, Mawatari T, Fukushi J, Oyamada A, Fujimura K, Iwamoto Y and Yoshikai Y: Preferential accumulation of activated Th1 cells not only in rheumatoid arthritis but also in osteoarthritis joints. J Rheumatol 38: 1569-1575, 2011.

26. Lùrati $\mathrm{A}$, Laria $\mathrm{A}$, Mazzocchi $\mathrm{D}$, Re KA, Marrazza $M$ and Scarpellini M: Effects of hyaluronic acid (HA) viscosupplementation on peripheral Th cells in knee and hip osteoarthritis. Osteoarthritis Cartilage 23: 88-93, 2015.

27. Kwok SK, Cho ML, Park MK, Oh HJ, Park JS, Her YM, Lee SY, Youn J, Ju JH, Park KS, et al: Interleukin-21 promotes osteoclastogenesis in humans with rheumatoid arthritis and in mice with collagen-induced arthritis. Arthritis Rheum 64: 740-751, 2012. 